\title{
Uma Proposta de Avaliação de Conceitos, Práticas e Perspectivas de Pensamento Computacional
}

\author{
Bianca L. Santana ${ }^{1}$, Christina F. G. Chavez ${ }^{1}$, Roberto A. Bittencourt ${ }^{2}$ \\ ${ }^{1}$ Universidade Federal da Bahia \\ Av. Adhemar de Barros, s/n, Ondina \\ Salvador - BA, Brasil - 40170-110 \\ ${ }^{2}$ UEFS - Universidade Estadual de Feira de Santana \\ Av. Transnordestina, $\mathrm{s} / \mathrm{n}$, Novo Horizonte \\ Feira de Santana - BA, Brasil - 44036-900 \\ biancasantana.ls@gmail.com, flach@ufba.br, roberto@uefs.br
}

\begin{abstract}
In this paper, we present a preliminary work of building assessment instruments of Computational Thinking for middle school students. Our proposal consists of two tests that consider the dimensions of concepts, practices and perspectives of Computational Thinking. We also present a proposal to assure instruments' validity and reliability based on psychometrics.

Resumo. Neste artigo, apresentamos o trabalho preliminar de construção de uma avaliação da aquisição do Pensamento Computacional para estudantes do Ensino Fundamental II. Nossa proposta de avaliação, composta por dois testes, considera as dimensões de conceitos, práticas e perspectivas de Pensamento Computacional. Também apresentamos uma proposta para garantir a validade e confiabilidade dos instrumentos com base na psicometria.
\end{abstract}

\section{Introdução}

Pensamento Computacional (PC) é um termo em destaque na comunidade de Educação em Computação desde 2006, com o trabalho de Jeannette Wing intitulado Computational Thinking [Wing 2006]. Desde então, PC tem sido considerado uma competência importante, presente em muitas experiências de Educação em Computação apresentadas na literatura, além de ser um eixo recorrente em currículos de Computação para escolas em diversos países, inclusive no Brasil [Seehorn et al. 2011, Lye e Koh 2014, SBC 2017, Hsu et al. 2018]. Consequentemente, a avaliação da aquisição do PC pelos estudantes também recebe destaque na área de Educação em Computação. As iniciativas propostas para avaliar o desempenho dos estudantes variam de acordo com a abordagem pedagógica, o objetivo da avaliação e a interpretação do conceito de PC [Araújo et al. 2016]. Por outro lado, a maioria dos instrumentos não avalia todos os aspectos do PC e carece de validação psicométrica [Cutumisu et al. 2019].

Neste artigo, apresentamos o trabalho preliminar de construção de uma avaliação de PC com foco na segunda etapa do Ensino Fundamental. Este trabalho considera o currículo Computação Fundamental ${ }^{1}$, desenvolvido para as séries do Ensino Fundamental II brasileiro.

\footnotetext{
${ }^{1} \mathrm{https}$ //sites.google.com/view/computacaofundamental/
} 


\section{O que é Pensamento Computacional}

Apesar do imenso destaque na comunidade de Educação em Computação, não há um consenso na literatura sobre o que significa exatamente PC e quais habilidades estão associadas a ele. Revisões da literatura que evidenciam os termos que mais aparecem nos trabalhos publicados apontam que PC é entendido como uma abordagem de solução de problemas que envolve habilidades como abstração, pensamento algorítmico, decomposição, avaliação e generalização [Selby e Woollard 2013]. As muitas definições operacionais disponíveis na literatura, dificultam o estabelecimento das abordagens que potencializam o PC [Barr et al. 2011, Grover et al. 2015].

Um dos frameworks de PC mais utilizados, desenvolvido por Brennan e Resnick (2012), define PC como uma abordagem para a resolução de problemas composta por três dimensões principais: Conceitos, Práticas e Perspectivas. Conceitos de PC são aqueles que os estudantes utilizam ou aprendem enquanto programam: sequências, loops, paralelismos, eventos, condicionais, operadores e dados. Práticas são aquelas que os estudantes desenvolvem à medida que se envolvem com os conceitos: ser incremental e iterativo, testar e depurar, reutilizar e remixar, e abstrair e modularizar. As perspectivas são criadas pelos estudantes sobre o mundo e sobre si mesmos à medida em que se envolvem com os conceitos e práticas: expressão, conexão e questionamento.

\section{Avaliação do Pensamento Computacional}

Identificamos três tipos principais de instrumentos de avaliação da aquisição do PC: provas de avaliação, frameworks para análise manual de projetos e ferramentas de análise automática de projetos. As subseções a seguir descrevem cada um dos tipos de avaliação.

\subsection{Provas de avaliação}

As provas de avaliação do PC distinguem-se em dois tipos básicos: as que procuram avaliar a aquisição de habilidades do PC, sem recorrer a habilidades concretas de programação, e as que consideram a programação, com questões e tarefas baseadas em construções de código em linguagens de programação visual ou textual.

De modo geral, iniciativas que buscam avaliar as habilidades fundamentais do PC sem recorrer a habilidades concretas de programação podem ser utilizadas para comparar diversas abordagens, e podem ser empregadas como pré- e pós-testes. Nesta categoria de instrumentos de avaliação, destaca-se o Bebras, um teste composto por perguntas de múltipla escolha e resposta curta organizado anualmente desde 2004 e que tem, como público-alvo, estudantes do ensino fundamental e médio [Dagiene e Stupurienè 2016].

Provas que exigem conhecimentos prévios em alguma linguagem de programação podem oferecer avaliações mais concretas para conceitos e práticas de PC. Por exemplo, o Teste de Pensamento Computacional (CTt) é um instrumento com itens de múltipla escolha, desenvolvido para estudantes da sétima e oitava séries do ensino fundamental, cujo objetivo principal é avaliar o PC, considerando a sintaxe e lógica das linguagens de programação, como sequências, loops, condicionais, funções e variáveis [RománGonzález et al. 2017].

Poucos instrumentos dedicam-se a avaliação de perspectivas de PC. O Programming Empowerement Survey, prova com 26 itens em escala de Likert, avalia atitudes do estudante em relação à programação como autoeficácia criativa, autoeficácia 
na programação, interesse e atitude em relação à colaboração [Kong et al. 2018]. Outro instrumento que avalia explicitamente perspectivas de PC é o Three-Dimensional Integrated Assessment (TDIA), que avalia a aquisição de conceitos, práticas e perspectivas através de alterações feitas em cenários pré-definidos no ambiente Alice [Zhong et al. 2016].

\subsection{Avaliação de projetos de programação}

Análise de projetos de programação é um recurso bastante utilizado para avaliação do PC, que pode ser feita manual ou automaticamente. A análise manual permite a contemplação de aspectos relacionados as práticas e perspectivas de PC, como a criatividade, difíceis de mensurar através da análise automática. Em contrapartida, ferramentas automáticas de avaliação destacam-se pela agilidade, permitindo a aplicação em larga escala.

O framework Progression of Early Computational Thinking (PECT) avalia a aquisição do PC nas séries iniciais do ensino fundamental por meio da análise manual de programas escritos em Scratch [Seiter e Foreman 2013]. O modelo avalia os seguintes conceitos de PC: Procedimentos e Algoritmos, Decomposição de Problemas, Paralelização e Sincronização, Abstração e Representação de Dados. O desenvolvimento de apps para análise do PC em projetos de Scratch também recebe destaque. A ferramenta Hairball faz análise automática de projetos no Scratch considerando quatro dimensões: inicialização de estado, sincronização de blocos de fala e arquivos de som, transmissão e recebimento de mensagens para desencadear ações e animação [Boe et al. 2013]. A ferramenta $D r$. Scratch é uma aplicação que permite analisar projetos Scratch, indicando habilidades de PC e oferecendo feedback com possíveis melhorias nos projetos, a fim de aumentar o nível de desenvolvimento do PC [Moreno-León et al. 2015].

\section{Computação Fundamental}

O projeto Computação Fundamental é um currículo para o Ensino Fundamental II, do sexto ao nono ano. Neste currículo, a Computação é um tópico regular de estudos com carga horária de 60 horas anuais, divididas em 30 aulas. Cada série possui um livro do professor, com orientações sobre as aulas, e o livro do estudante, com textos e exercícios.

O currículo é baseado nos cinco eixos temáticos e resultados de aprendizagem apresentados no currículo da CSTA: Pensamento Computacional; Práticas de Computação e Programação; Colaboração; Computadores e Dispositivos de Comunicação; e Impactos Comunitários, Globais e Éticos [Seehorn et al. 2011]. Cada um dos eixos possui uma série de tópicos que devem ser cumpridos.

O programa do sexto ano, intitulado Computação $\boldsymbol{e} \boldsymbol{E} \boldsymbol{u}$, trabalha com temas como representação binária, álgebra booleana, uso do computador, programação com tutoriais do Code.org e criação de animações em Scratch [Santana et al. 2019a, Santana et al. 2019b]. O programa do sétimo ano, intitulado Computação e Comunidade, trabalha com o desenvolvimento de jogos e programas diversos no Scratch, colaboração através da comunidade online do Scratch, busca, inteligência artificial, autômatos finitos, dentre outros [Araujo et al. 2019a, Araujo et al. 2019b]. No programa do oitavo ano, intitulado Computação e Sociedade, os estudantes aprendem sobre redes de computadores, colaboração através das ferramentas online da Google, questões éticas relacionadas ao compartilhamento de informações online, robótica com o robô $m B o t$ e a ferramenta mBlock da empresa Makeblock, programação de edição de imagens, através da ferramenta 
GP Blocks, e a linguagem de programação Python e com o tema desenhos de figuras geométricas através da biblioteca Turtle [Santana et al. 2020]. O programa do nono ano, intitulado Computação e o Mundo, trabalha com o desenvolvimento de simulações de fenômenos da ciência através do Scratch, programação em Python no contexto de manipulação de imagens e programas simples, e hardware livre através da plataforma Arduino [Araujo et al. 2020].

\section{Metodologia}

Esta fase preliminar do trabalho envolveu três tarefas: proposição da definição operacional de PC; definição do sistema de avaliação e elaboração das tabelas de avaliação; e a proposta de validação dos instrumentos.

Definição operacional. A definição operacional foi escolhida após uma ampla revisão de literatura e estudo sobre o currículo Computação Fundamental. Nesta etapa identificamos as principais habilidades associadas ao PC, que nos permitiu selecionar as habilidades que serão avaliadas.

Proposição dos instrumentos de avaliação. Através da análise de instrumentos já existentes, identificamos as principais lacunas para avaliação de PC. A partir disso, definimos nossa proposta de avaliação.

Proposta de validação. Nesta etapa, elaboramos a proposta prévia de validação para toda a abordagem de avaliação apresentada e seus respectivos instrumentos. Examinamos os aspectos psicométricos necessários para a validação de testes, além de trabalhos de validação de instrumentos relacionados à aprendizagem de programação [American Educational Research Association et al. 2014, Tew e Guzdial 2011, Wiebe et al. 2003]. A partir destas análises, definimos uma proposta preliminar que verifica o conteúdo e finalidade da nossa avaliação.

\section{Resultados}

Descrevemos, a seguir, os resultados preliminares que consistem na definição operacional de PC adotada, proposta de avaliação do PC e a respectiva proposta de validação.

\subsection{Definição operacional de PC}

As habilidades de PC selecionadas estão expressas na Figura 1. Neste trabalho, consideramos o framework desenvolvido por Brennan e Resnick (2012) e o conceito de PC adotado pelo CSTA [Brennan e Resnick 2012, Seehorn et al. 2011]. Também consideramos os resultados de aprendizagem definidos no currículo Computação Fundamental.

Consideramos que Pensamento Computacional é uma abordagem para a resolução de problemas que envolve habilidades separadas entre três dimensões-chave: conceitos, práticas e perspectivas. As práticas de $\mathrm{PC}$ se desenvolvem à medida em que os estudantes se envolvem com os conceitos, e as perspectivas são uma dimensão que envolve habilidades relacionadas à visão do estudante sobre a computação e suas possibilidades.

Nosso sistema de avaliação pressupõe que o Currículo Computação Fundamental trabalha o PC nos eixos de conceitos, práticas e perspectivas. Consequentemente, nossa visão em relação às habilidades de PC é mais ampla do que a definição considerada pelos autores do CSTA. Deste modo, os resultados de aprendizagem do currículo Computação 
Fundamental nos eixos de Pensamento Computacional, Práticas de Computação e Programação e Colaboração contribuem para o desenvolvimento de habilidades de PC.

\begin{tabular}{|c|c|c|}
\hline Conceitos & Práticas & Perspectivas \\
\hline Pensamento Algoritmico & Iteração & $\begin{array}{l}\text { Expressão } \\
\text { E1: Eu posso criar artefatos com- }\end{array}$ \\
\hline Sequências & Abstração & $\begin{array}{l}\text { putacionais } \\
\text { E2: Eu posso expressar minhas } \\
\text { ideias através da computação }\end{array}$ \\
\hline Repetições & & \\
\hline Condicionais & Modularização & $\begin{array}{l}\text { Conexão } \\
\text { C1: Eu posso colaborar com outras } \\
\quad \text { pessoas }\end{array}$ \\
\hline Operadores & Generalização & $\begin{array}{l}\text { C2: Eu posso criar soluções para } \\
\text { outras pessoas }\end{array}$ \\
\hline & & Questionamento \\
\hline Paralelismo & $\begin{array}{l}\text { Processamento e análise de } \\
\text { dados }\end{array}$ & $\begin{array}{l}\text { Q1: Eu posso questionar para } \\
\text { entender artefatos e soluções } \\
\text { computacionais }\end{array}$ \\
\hline Eventos & Teste e depuração & $\begin{array}{l}\text { Q2: Eu posso alterar artefatos e } \\
\text { soluções computacionais já } \\
\text { existentes }\end{array}$ \\
\hline
\end{tabular}

Figura 1. Framework conceitual adotado.

\subsection{Avaliação}

A avaliação proposta verifica a aquisição do PC com base na definição operacional adotada. Nosso objetivo é identificar a aquisição dos conceitos, práticas e perspectivas pertencentes ao PC e o nível em que esta ocorre. Para isso, propomos a elaboração de dois instrumentos: um para medir conceitos e práticas e o outro para medir perspectivas. Ambos os instrumentos devem ser aplicados ao final do currículo, tendo como públicoalvo jovens que estejam no nono ano do Ensino Fundamental. A seguir, descrevemos os instrumentos propostos.

\subsubsection{Avaliando conceitos e práticas de PC}

Para avaliar a aquisição de conceitos e práticas de $\mathrm{PC}$, propomos a elaboração de um instrumento especifico, cujos construtos envolvem os conceitos e práticas listados na Figura 1. As questões deste instrumento podem envolver explicitamente linguagens de programação em bloco ou textuais. $\mathrm{O}$ instrumento deve ser aplicado com estudantes que já possuem algum conhecimento sobre programação de computadores.

Todas as questões serão de múltipla escolha, onde apenas uma alternativa está correta. Em cada item, as alternativas de resposta podem ser apresentadas em qualquer um destes três estilos: trechos de código (em bloco ou textual), resultado final após a execução do programa ou lista de ações. Todas as questões trarão algum tipo de representação através de figuras ou trechos de código como forma de aumentar a discriminação e ajudar a representação mental do leitor.

As questões podem ser de dois tipos distintos: avaliam conceitos isoladamente, ou avaliam a combinação de conceitos que culminam em alguma das práticas de PC listadas. Deste modo, haverá dependência direta entre o conhecimento de um conceito e a aplicação de práticas a ele associadas. O instrumento deve ter três questões para cada conceito e prática de PC. Cada item avalia se o estudante apresenta uma determinada habilidade e o nível em que isto ocorre: básico, intermediário ou proficiente. 
As definições de nível foram feitas considerando como as habilidades de PC e programação se relacionam com o domínio cognitivo da taxonomia de Bloom [Selby 2015]. As questões que avaliam apenas conceitos de PC consideram: nível básico, quando o estudante demonstra a capacidade de reconhecer e compreender determinado conceito; nível intermediário, quando o estudante é capaz de aplicar o conceito; e nível proficiente, quando o estudante é capaz de criar estruturas mais complexas empregando o conceito e avaliando a efetividade da solução. Questões que avaliam as práticas de PC consideram: nível básico, quando o estudante é capaz de identificar determinado padrão; nível intermediário, quando o estudante é capaz de identificar a necessidade de uso da prática e de elaborar uma solução simples; e nível proficiente, quando o estudante é capaz de escolher entre diferentes estratégias de implementação para determinada prática.

\subsubsection{Avaliando perspectivas}

Para avaliar as perspectivas de $\mathrm{PC}$, propomos um instrumento baseado em cenários. $\mathrm{O}$ instrumento será composto por seis cenários distintos acompanhados de questões de múltipla escolha. Este instrumento também pressupõe que os estudantes possuem conhecimentos sobre programação.

Os cenários ainda estão em fase de concepção inicial, mas a Figura 2 descreve as características principais que cada cenário deve ter. Cada cenário mede um aspecto diferente, de modo que todas as perspectivas previstas são protagonistas em um cenário. Um cenário pode descrever uma situação do mundo real que pode ser tratada pela Computação, um programa de computador ou um dispositivo que envolve eletrônica e robótica. A intenção é que o estudante sinta-se imerso em uma experiência específica relacionada à Computação e indique quais atitudes tomar a partir dela.

\begin{tabular}{|c|c|c|}
\hline $\begin{array}{l}\text { Cenário e } \\
\text { habilidade }\end{array}$ & Objetivo & Tipo de cenário \\
\hline $\begin{array}{l}\text { Cenário } 1 \\
\text { E1 }\end{array}$ & $\begin{array}{l}\text { Identificar se os estudantes sentem-se capazes de } \\
\text { implementar os artefatos propostos, empregando } \\
\text { conhecimentos que já possuem ou buscando novos } \\
\text { conhecimentos. }\end{array}$ & $\begin{array}{l}\text { Descreve a ideia de um programa } \\
\text { ou um programa já existente. }\end{array}$ \\
\hline $\begin{array}{l}\text { Cenário } 2 \\
\text { E2 }\end{array}$ & $\begin{array}{l}\text { Identificar se os estudantes são capazes de apontar a } \\
\text { criação de artefatos computacionais como um meio } \\
\text { efetivo e poderoso para a expressão e criatividade. }\end{array}$ & $\begin{array}{l}\text { Descreve uma situação do mundo } \\
\text { real que pode ser tratada pela } \\
\text { computação. }\end{array}$ \\
\hline $\begin{array}{l}\text { Cenário } 3 \\
\text { C1 }\end{array}$ & $\begin{array}{l}\text { Identificar se os estudantes apontam maneiras pelas } \\
\text { quais a colaboração pode ser utilizada para criar } \\
\text { artefatos computacionais. }\end{array}$ & $\begin{array}{l}\text { Descreve a ideia ou um projeto já } \\
\text { existente de programação ou } \\
\text { eletrônica/robótica. }\end{array}$ \\
\hline $\begin{array}{l}\text { Cenário } 4 \\
\text { C2 }\end{array}$ & $\begin{array}{l}\text { Identificar se os estudantes expressam a possibilidade } \\
\text { de engajar, entreter, educar ou equipar outras pessoas } \\
\text { através de suas criações computacionais. }\end{array}$ & $\begin{array}{l}\text { Descreve uma situação do mundo } \\
\text { real que pode ser tratada pela } \\
\text { computação. }\end{array}$ \\
\hline $\begin{array}{l}\text { Cenário } 5 \\
\text { Q1 }\end{array}$ & $\begin{array}{l}\text { Identificar se os estudantes apresentam confiança para } \\
\text { associar suas habilidades com tecnologias existentes e } \\
\text { questioná-las. }\end{array}$ & $\begin{array}{l}\text { Descreve uma situação do mundo } \\
\text { real que pode ser tratada pela } \\
\text { computação. }\end{array}$ \\
\hline $\begin{array}{l}\text { Cenário } 6 \\
\text { Q2 }\end{array}$ & $\begin{array}{l}\text { Identificar se os estudantes apresentam confiança para } \\
\text { associar suas habilidades com as tecnologias existentes } \\
\text { e alterá-las. }\end{array}$ & $\begin{array}{l}\text { Descreve a ideia ou um projeto já } \\
\text { existente de programação ou } \\
\text { eletrônica/robótica. }\end{array}$ \\
\hline
\end{tabular}

Figura 2. Características da avaliação de perspectivas em PC.

Nos cenários dedicados à perspectiva de Expressão, buscamos indicadores de que os estudantes se sentem capazes de expressar sua criatividade de maneira ativa com a 
Computação. Nos cenários dedicados à perspectiva de conexão, buscamos indicadores de que os estudantes entendem a comunicação e colaboração com os outros como elementos atrelados a Computação. Com os cenários que avaliam a perspectiva de questionamento, buscamos indicadores de que os estudantes estabelecem conexão entre as suas habilidades com a Computação e a capacidade de compreender e aperfeiçoar o mundo tecnológico.

\subsection{Proposta de validação dos instrumentos}

O desenvolvimento dos instrumentos de avaliação propostos seguirá um processo iterativo iniciado com a especificação e seguido pela verificação do conteúdo e da finalidade do exame. A primeira etapa de validação prevista consiste na revisão por especialistas da proposta inicial, incluindo a definição operacional de PC e a especificação dos instrumentos. O uso de especialistas na etapa inicial de construção dos instrumentos tem como objetivo garantir a construção cuidadosa do teste. Apenas após esta etapa, seguiremos para a construção da primeira versão dos instrumentos de avaliação.

Para estabelecer a validade e a confiabilidade dos instrumentos, comporemos um painel de especialistas como meio de validação para o conteúdo dos testes. O painel de especialistas deve avaliar se os itens são inteligíveis e se os itens constituem uma representação adequada dos fatores [Pasquali 2011]. Ao avaliar a inteligibilidade, os especialistas verificam se o enunciado do problema pode ser compreendido e se este não apresenta elementos que atrapalham o entendimento por parte do estudante. Os especialistas também devem avaliar se os itens constituem uma representação adequada dos fatores, ou seja, se referem-se ou não ao conceito, prática ou perspectiva de PC que se deseja medir.

Em seguida, propomos um estudo piloto, onde os instrumentos serão aplicados com uma amostra do público alvo. $\mathrm{Na}$ análise empírica dos itens, pretendemos verificar a dificuldade, discriminação e a tendenciosidade de resposta, ou seja, se é possível ou não acertar as respostas apenas "chutando". Para isso, promoveremos análises estatísticas pautadas pela Teoria da Resposta ao Item (TRI), que é considerada uma abordagem prática e robusta para projetar instrumentos de avaliação de PC com itens dicotômicos ou de múltipla escolha [Araújo et al. 2020].

A partir dos resultados das análises com a TRI, os instrumentos serão melhorados, gerando uma nova versão. Um estudo será conduzido, aplicando a nova versão dos instrumentos com uma amostra maior do público-alvo e repetindo as análises estatísticas baseadas na TRI.

\section{Discussão}

Nossa abordagem para avaliação do PC explora algumas lacunas na literatura. A primeira delas é propor uma avaliação originalmente em português para um currículo completo do Ensino Fundamental II, também desenvolvido em português e aplicado no cenário nacional. Isto representa avanços significativos na pesquisa em âmbito nacional, uma vez que a falta de experiências replicáveis e o uso de instrumentos validados é apontada como uma lacuna neste cenário [Araujo et al. 2016, Santos et al. 2018].

Muitas avaliações se propõem a investigar conceitos e práticas, mas poucas consideram as perspectivas em CT [Cutumisu et al. 2019]. Nossa proposta considera uma definição operacional de PC com conceitos, práticas e perspectivas que combina definições importantes na literatura [Seehorn et al. 2011, Brennan and Resnick 2012]. 
Neste contexto, os dois instrumentos propostos, oferecem uma avaliação robusta do PC. Outro fator importante é a possibilidade de, após a validação dos instrumentos, examinar relações entre as dimensões do PC e resultados de aprendizagem em outras disciplinas, além de potenciais diferenças de gênero, variáveis sociais e demográficas, dentre outras.

Nossa proposta de validação explora evidências psicométricas e procura seguir metodologias consolidadas, como o uso de painel de especialistas e TRI. Esta é uma lacuna apontada na literatura, uma vez que poucas experiências reportam evidências psicométricas, como confiabilidade e validade, ou aplicação extensiva em larga escala [Cutumisu et al. 2019].

\section{Conclusões}

Neste trabalho, apresentamos uma proposta de avaliação da aquisição do PC. Consideramos uma definição operacional ampla de PC, com as dimensões de conceitos, práticas e perspectivas. Dois instrumentos serão elaborados, um instrumento específico para avaliação de conceitos e práticas, e outro específico para avaliação de perspectivas.

Como resultados preliminares, apresentamos a definição operacional empregada, a proposta de avaliação e a proposta de validação dos instrumentos. Os instrumentos poderão ser aplicados com estudantes do nono ano do Ensino Fundamental II, que tenham passado pela experiência de fomento ao PC envolvendo a programação de computadores, principalmente o Currículo Computação Fundamental. Para validação dos instrumentos, propomos o uso de painel de especialistas e TRI. Acreditamos que nossa proposta de avaliação explora lacunas na literatura, como a falta de avaliações que considerem todos as dimensões do PC, instrumentos com evidências psicométricas de validação e a falta de trabalhos completos no âmbito nacional [Cutumisu et al. 2019, Araújo et al. 2016, Araújo et al. 2016].

Em trabalhos futuros, organizaremos um painel de especialistas para validar a definição operacional de PC adotada e a proposta de avaliação. Seguiremos para a elaboração da primeira versão dos instrumentos, e sua validação por parte de um novo painel de especialistas. Conduziremos um estudo piloto, aplicando os instrumentos em uma amostra do público alvo e validando-os através da TRI. A partir dos resultados do estudo piloto, promoveremos alterações nos instrumentos, conduzindo um novo estudo, com a subsequente aplicação do instrumento e validação por TRI.

\section{Referências}

American Educational Research Association, American Psychological Association, Joint Committee on Standards for Educational, Psychological Testing (US), \& National Council on Measurement in Education. (2014). Standards for Educational and Psychological Testing. American Educational Research Association.

Araújo, A. L., Andrade, W., \& Guerrero, D. (2016). Um mapeamento sistemático sobre a avaliação do pensamento computacional no Brasil. In Anais dos Workshops do Congresso Brasileiro de Informática na Educação, volume 5, page 1147.

Araújo, A. L. S. O., Santos, J. S., Melo, M. R. A., Andrade, W. L., Guerreiro, D. D., \& Figueiredo, J. C. A. (2020). Teoria de resposta ao item. In Jaques, P. A., Pimentel, M., Siqueira, S., \& Bittencourt, I., editores, Metodologia de Pesquisa em Informática na Educação: Abordagem Quantitativa de Pesquisa, capítulo 8. SBC, Porto Alegre. 
Araújo, A. L. S. O., Andrade, W. L., \& Serey Guerrero, D. D. (2016). A systematic mapping study on assessing computational thinking abilities. In 2016 IEEE Frontiers in Education Conference (FIE), pages 1-9.

Araujo, L. G. J., Santana, B. L., \& Bittencourt, R. A. (2019a). Computação e Comunidade: Uma Proposta de Educação em Computação para o Sétimo Ano do Ensino Fundamental II. In Anais do XXV Workshop de Informática na Escola (WIE 2019).

Araujo, L. G. J., Santana, B. L., \& Bittencourt, R. A. (2019b). Computação e Comunidade: Livro do Professor. Edição do Autor, Feira de Santana. https://sites.google.com/view/computacaofundamental/setimoano.

Araujo, L. G. J., Santana, B. L., \& Bittencourt, R. A. (2020). Computação e o Mundo: Livro do Professor. Edição do Autor, Feira de Santana. https://sites.google.com/view/computacaofundamental/nonooano.

Barr, D., Harrison, J., \& Conery, L. (2011). Computational thinking: A digital age skill for everyone. Learning \& Leading with Technology, 38(6):20-23.

Boe, B., Hill, C., Len, M., Dreschler, G., Conrad, P., \& Franklin, D. (2013). Hairball: Lint-inspired static analysis of Scratch projects. In Proceedings of the 44th ACM Technical Symposium on Computer Science Education, SIGCSE '13, pages 215-220, New York, NY, USA. ACM.

Brennan, K. \& Resnick, M. (2012). New frameworks for studying and assessing the development of computational thinking. In Proceedings of the 2012 Annual Meeting of the American Educational Research Association, Vancouver, Canada, pages 1-25.

Cutumisu, M., Adams, C., \& Lu, C. (2019). A scoping review of empirical research on recent computational thinking assessments. Journal of Science Education and Technology, 28(6):651-676.

Dagiene, V. \& Stupurienè, G. (2016). Bebras - a sustainable community building model for the concept based learning of informatics and computational thinking. Informatics in Education, 15:25-44.

Grover, S., Pea, R., \& Cooper, S. (2015). Designing for deeper learning in a blended computer science course for middle school students. Computer Science Education, 25(2):199-237.

Hsu, T.-C., Chang, S.-C., \& Hung, Y.-T. (2018). How to learn and how to teach computational thinking: Suggestions based on a review of the literature. Computers \& Education, 126:296-310.

Kong, S.-C., Chiu, M. M., \& Lai, M. (2018). A study of primary school students' interest, collaboration attitude, and programming empowerment in computational thinking education. Computers \& Education, 127:178-189.

Lye, S. Y. \& Koh, J. H. L. (2014). Review on teaching and learning of computational thinking through programming: What is next for K-12? Computers in Human Behavior, 41:51-61.

Moreno-León, J., Robles, G., \& Román-González, M. (2015). Dr. Scratch: Automatic analysis of Scratch projects to assess and foster computational thinking. RED-Revista de Educación a Distancia. 
Pasquali, L. (2011). Psicometria: Teoria dos Testes na Psicologia e na Educação. Vozes.

Román-González, M., Pérez-González, J.-C., \& Jiménez-Fernández, C. (2017). Which cognitive abilities underlie computational thinking? Criterion validity of the Computational Thinking test. Computers in Human Behavior, 72:678-691.

Santana, B. L., Araujo, L. G. J., \& Bittencourt, R. A. (2019a). Computação e Eu: Uma Proposta de Educação em Computação para o Sexto Ano do Ensino Fundamental II. In WEI 2019 - XXVII Workshop sobre Educação em Computação.

Santana, B. L., Araujo, L. G. J., \& Bittencourt, R. A. (2019b). Computação e Eu: Livro do Professor. Edição do Autor, Feira de Santana. https://sites.google.com/view/computacaofundamental/sextoano.

Santana, B. L., Araujo, L. G. J., \& Bittencourt, R. A. (2020). Computação e Sociedade: Livro do Professor. Edição do Autor, Feira de Santana. https://sites.google.com/view/computacaofundamental/oitavoano.

Santos, P. S., Araujo, L. G. J., \& Bittencourt, R. A. (2018). A Mapping Study of Computational Thinking and Programming in Brazilian K-12 Education. In 2018 IEEE Frontiers in Education Conference (FIE). IEEE.

SBC (2017). Referenciais de Formação em Computação: Educação Básica. http://www.sbc.org.br/files/ComputacaoEducacaoBasica-versaofinal-julho2017.pdf.

Seehorn, D., Carey, S., Fuschetto, B., Lee, I., Moix, D., O’Grady-Cunniff, D., Owens, B. B., Stephenson, C., \& Verno, A. (2011). CSTA K-12 Computer Science Standards: Revised 2011. Technical report, CSTA/ACM, New York, NY, USA. 104111.

Seiter, L. \& Foreman, B. (2013). Modeling the learning progressions of computational thinking of primary grade students. In Proceedings of the Ninth Annual International ACM Conference on International Computing Education Research, ICER '13, pages 59-66, New York, NY, USA. ACM.

Selby, C. \& Woollard, J. (2013). Computational Thinking: The Developing Definition. University of Southampton (E-prints).

Selby, C. C. (2015). Relationships: Computational thinking, pedagogy of programming, and Bloom's taxonomy. In Proceedings of the Workshop in Primary and Secondary Computing Education, WiPSCE '15, pages 80-87, New York, NY, USA. ACM.

Tew, A. E. \& Guzdial, M. (2011). The FCS1: A language independent assessment of CS1 knowledge. In Proceedings of the 42nd ACM Technical Symposium on Computer Science Education, SIGCSE '11, pages 111-116, New York, NY, USA. ACM.

Wiebe, E., Williams, L. A., Yang, K., \& Miller, C. S. (2003). Computer Science Attitude Survey. Technical report, North Carolina State University. Dept. of Computer Science.

Wing, J. M. (2006). Computational thinking. Communications of the ACM, 49(3):33-35.

Zhong, B., Wang, Q., Chen, J., \& Li, Y. (2016). An exploration of three-dimensional integrated assessment for computational thinking. Journal of Educational Computing Research, 53(4):562-590. 